\title{
Electron-Capture Dissociation
}

National Cancer Institute

\section{Source}

National Cancer Institute. Electron-Capture Dissociation. NCI Thesaurus. Code C161782.

A method to induce ion fragmentation through the introduction of low-energy electrons to ions trapped in a gaseous phase. 